\title{
DISEÑO, CONSTRUCCIÓN Y SISTEMATIZACIÓN DE UNA ESTACIÓN METEOROLÓGICA DE REGISTRO AUTOMÁTICO Y PROCESAMIENTO DE TRES PARÁMETROS BASADO EN LA IMPLEMENTACIÓN DE HARDWARE LIBRE
}

\section{DESIGN, CONSTRUCTION AND SYSTEMATIZATION OF A METEOROLOGICAL STATION OF AUTOMATIC REGISTRATION AND THREE-PARAMETER PROCESSING BASED ON FREE HARDWARE IMPLEMENTATION}

\author{
Carlos Polo Bravo, Hugo Torres Muro, Juan Ríos \\ Marquezado, Rigoberto Bernabé Coaquera, \\ Alessandro De La Gala Contreras ${ }^{5}$
}

\section{RESUMEN}

En el presente trabajo trata sobre el diseño, construcción y sistematización de una estación meteorológica para el registro automático, procesamiento y monitoreo electrónico de tres parámetros meteorológicos: temperatura ambiente, irradiancia solar global y humedad relativa ambiental, conprecisiones de $0,1^{\circ} \mathrm{C} ; 0,1$ watts $/ \mathrm{m} 2$ y de $1 \%$, respectivamente, usando el hardware libre denominado ARDUINO; el sistema monitorea, registra, almacena información en forma automática de acuerdo a la programación pre establecida por el algoritmo y transfiere información a una computadora para el tratamiento correspondiente.

Para el efecto se ha utilizado componentes, materiales y sensores de bajo costo y buena funcionalidad, para medir la irradiancia solar es del tipo LDR (fotoresistor) y el DHT11 para medir la humedad relativa y temperatura ambiente, que se han adquirido en el mercado local y nacional; y para el sistema que protege a los sensores de humedad relativa y temperatura ambiente se ha reusado una carcasa de una antigua estación meteorológica, el costo total del sistema es de S/ 347,00 que comparado con el costo de las estaciones meteorológicas importadas resulta ser inferior en un factor de 100 a 200 veces. La información registrada es tan igual que la que proporciona cualquier estación meteorológica importada de las mejores marcas y modelos, puede ser muy útil para un estudio científico y predicción de diferentes fenómenos meteorológicos y desastres naturales.

Palabras claves: estación meteorológica, hardware Arduino, sensores, temperatura y humedad relativa ambiente, irradiancia solar

\section{ABSTRACT}

The present work deals with the design, construction and systematization of a meteorological station for the automatic recording, processing and electronic monitoring of three meteorological parameters: ambient temperature, global solar irradiance and ambient relative humidity, with precision of $0.1^{\circ} \mathrm{C} ; 0.1$ watts / $\mathrm{m} 2$ and $1 \%$, respectively, using the free hardware called ARDUINO; the system automatically monitors, registers, stores information according to the pre-established scheduling algorithm and transfers information to a computer for the corresponding processing.

For this purpose, low-cost components, materials and sensors have been used to measure the solar irradiance of the type LDR (photoresistor) and DHT11 to measure relative humidity and ambient temperature, which have been acquired in the local market and national; and for the system that protects the sensors of relative humidity and ambient temperature has been reused a housing of an old meteorological station, the total cost of the system is of $S / 347,00$ that compared to the cost of the imported meteorological stations turns out to be lower by a factor of 100 to 200 times. The recorded information is just as much as that provided by any weather station imported from the best makes and models, can be very useful for a scientific study and prediction of different meteorological phenomena and natural disasters.

\footnotetext{
Msc. con mención en Gestión Ambiental y Desarrollo Sostenible

Docente de la Facultad de Ciencias - Universidad Nacional Jorge Basadre Grohmann - Tacna Perú

2 Msc. con mención en Gestión Ambiental y Desarrollo Sostenible

Docente de la Facultad de Ciencias - Universidad Nacional Jorge Basadre Grohmann - Tacna Perú

3 Ingeniero de Minas

Docente de la Facultad de Ciencias - Universidad Nacional Jorge Basadre Grohmann - Tacna Perú

4 Licenciado en Física Aplicada

Docente de la Facultad de Ciencias - Universidad Nacional Jorge Basadre Grohmann - Tacna Perú

4 Bachiller en Física Aplicada con mención en Energías Renovables

Docente de la Facultad de Ciencias - Universidad Nacional Jorge Basadre Grohmann - Tacna Perú
} 


\section{INTRODUCCIÓN}

Hoy en día en todos los países del mundo, los científicos especialistas predicen con días de anticipación el estado del tiempo a través de diferentes métodos y usando diferentes tecnologías con fines ambientales, agrícolas, energéticos y del cambio climático.La disciplina encargada de la investigación de lo indicado es la Meteorología. En nuestro país y en Tacna, la institución responsable del estudio de los parámetros meteorológicos es el Servicio Nacional de Meteorología e Hidrografía (SENAMHI) que registra y trata tales parámetros en forma puntual en todas las regiones del país básicamente a través de tomas de datos en forma mecánica, y parcialmente en forma automatizada.

Por otro lado en la ciudad de Tacna, el Centro de Energías Renovables de Tacna (CERT) de la UNJBG cuenta con una estación meteorológica automatizada que registra y almacena continuamente los parámetros meteorológicos de temperatura y humedad relativa ambiental, velocidad y dirección del viento, irradiancia solar global, presión atmosférica, precipitación fluvial, brillo solar, entre otros que requieren ser tratadas científicamente para determinar su relación con la climatología local, el estado del tiempo, con el cambio climático y la salud poblacional; con el inconveniente que son altamente costosas, se tienen que importar. Nuestra región y el país requiere contar por lo menos en cada centro poblado o distrito con una estación meteorológica completa que brinde información en tiempo real y que se puedan estudiar científicamente los diferentes fenómenos naturales propios de la región, que adicionando otros estudios se puedan predecirlos oportunamente con fines de contingencias, adaptación y mitigación.

El trabajo de investigación experimental abarca el campo del medio ambiente, específicamente en lo referente al estudio meteorológico parcial de la ciudad de Tacna, para tal objetivo en esta primera etapa de un proyecto mayor e integral se ha diseñado y construido una estación meteorológica para el registro automático y procesamiento de tres parámetros meteorológicos: temperatura y humedad relativa ambiente e irradiancia solar global sobre superficie horizontal, el cual se ha ejecutado en el periodo de un año.
En la actualidad en la ciudad de Tacna y considerando los factores meteorológicos propios de la localidad, no se ha realizado o publicado de forma seria un estudio que proyecte el comportamiento de algunas variables meteorológicas a través del diseño, construcción y caracterización de una estación meteorológica con sensores modernos de bajo costo y software libre. Esto se debe al hecho de escasez de estaciones ya que son mayormente importadas, de elevado costo que no permite que sean muy comerciales o masificadas, a ello se suma que se cuenta con muy pocos profesionales especialistas en la materia.

El objetivo general es el diseño, construcción y sistematización de una estación meteorológica de registro automático y procesamiento de tres parámetros basado en la implementación de hardware libre (Arduino).

\section{ASPECTOS TEÓRICOS}

\section{ENERGÍA SOLAR Y SUS CARACTERÍSTICAS}

La energía proveniente del sol que llega a la superficie terrestre es bajo cuatro formas:

Irradiancia directa: Es aquella que llega directamente del Sol sin haber sufrido cambio alguno en su dirección.

Irradiancia difusa: Es aquella que proviene de la reflexión de las nubes, partículas de polvo atmosférico, montañas, árboles, edificios, el propio suelo, etc. y viaja en todas direcciones.

Irradiancia reflejada: La radiación reflejada es, como su nombre indica, aquella reflejada por la superficie terrestre. La cantidad de radiación depende del coeficiente de reflexión de la superficie, también llamado albedo.

Irradiancia global: Es la irradiancia total, es decir la suma de las irradiancias directa y la difusa.

La luz visible se extiende desde 0,4 a $0,74 \mu \mathrm{m}$. La radiación ultravioleta $u$ ondas cortas desde los 0,15 a $0,4 \mu \mathrm{m}$. y la radiación infrarroja $u$ ondas largas desde las 0,74 a $4 \mu \mathrm{m}$. Estas irradiancias, sobre la superficie terrestre se miden con ciertos equipos denominados piranómetros, heliógrafos y pirheliómetros, los cuales basan su funcionamiento en sensores cuya propiedad física varía con la irradiancia solar incidente, los más usados: los termoeléctricos, los fotovoltaicos, los de termópilas, los fotorresistores. 


\section{HARDWARE LIBRE}

Se llama hardware libre, al hardware de código abierto, electrónica libre o máquinas libres a aquellos dispositivos de hardware cuyas especificaciones y diagramas esquemáticos son de acceso público, ya sea bajo algún tipo de pago, $\circ$ de forma gratuita. La filosofía del software libre es aplicable a la del hardware libre, y por eso forma parte de la cultura libre.

El hardware libre (también llamado "open-source" o "de fuente abierta") comparte muchos de los principios y metodologías del software libre. En particular, el hardware libre permite que la gente pueda estudiarlo para entender su funcionamiento, modificarlo, reutilizarlo, mejorarlo y compartir dichos cambios.

\section{ESTACIÓN METEOROLÓGICA}

El estudio de los parámetros meteorológicos para estudiar el clima y el tiempo de una determinada zona geográfica, se realiza a través de las denominadas estaciones meteorológicas, las mediciones se realizan en lugares establecidos, donde es necesario contar con datos meteorológicos para fines diversos en tiempo real 0 en tiempo diferidos 0 ambos. Estos sistemas deben de contar con determinadas condiciones técnicas normalizadas, disponen de varios instrumentos o todos si es muy completa, se instalan bajo las normas establecidas por la Organización Meteorológica Mundial (OMM). Para que los datos sean rigurosos, en el recinto de una estación meteorológica debe disponer de una garita, una especie de jaula de madera blanca situada a $1,5 \mathrm{~m}$ del suelo, dentro de la cual se ubican los termómetros, el higrómetro y el evaporímetro. Además, en muchos casos, las estaciones disponen de una torre meteorológica, sobre esta se sitúan aparatos de medida como termómetros, anemómetros y veletas, que nos informan sobre las condiciones meteorológicas a distintas alturas. Las observaciones meteorológicas se realizan con diferentes fines como para realizar pronósticos y alertas sobre la ocurrencia de fenómenos severos, para ciertas operaciones locales, para pronóstico hidrológico y agro meteorológico así como para investigación en ciencias de la atmosfera.

Las estaciones meteorológicas automáticas están basadas en microprocesadores 0 microcomputadoras, y controladas por elementos de programación, son máquinas altamente adaptables, de gran salida de información, muy avanzadas respecto del sistema manual en velocidad y calidad. Los sensores usados son para medir la velocidad del viento: (anemómetro de cazoleta/interruptor de haz luminoso); precipitación (cangilón basculante), temperatura (termómetro de resistencia o termistores); dirección del viento: (veleta / potenciómetro de tres tomas); irradiancia solar (piranómetros, perheliómetros), radiación ultravioleta (UV), etc.

\section{MATERIALES Y MÉTODO \\ DISEÑO DE LA ESTACIÓN METEOROLÓGICA}

En esta parte se ha considerado que el sistema debe de tener una estructura sólida de metal o plástico en la cual pueda sujetar a cada uno de los sensores de la estación, cables de conexión, tarjeta de registro de datos, fuente de alimentación de energía, tarjeta arduino, reloj calendario, etc. de modo tal que se ha diseñado una caja porta el sensor LDR (carcasa) y su respectivo circuito electrónico, cuya forma y dimensiones se pueden visualizar en las figuras siguientes:

\section{Sensores}

El sensor digital de Temperatura DS18B20 de MAXIM a prueba de agua, permite conocer la temperatura utilizando solo 1 cable para datos.

Fig. 1: Sensor de temperatura

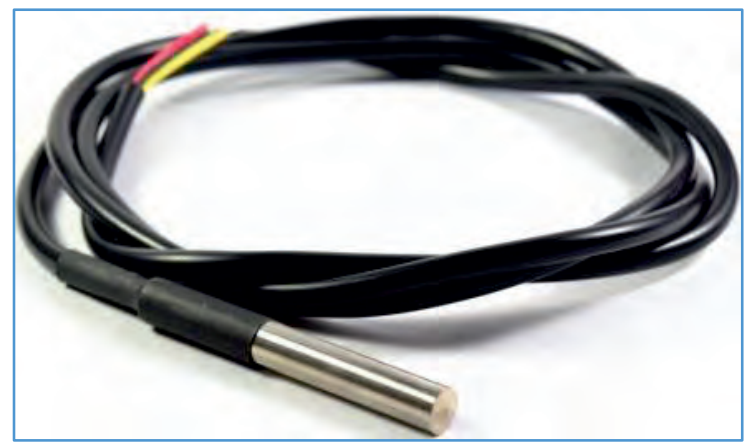

Una celda fotocondutora de CdS o LDR, es un dispositivo cuya resistencia varía de acuerdo con la cantidad de luz que reciba; este sensor fue adaptado para obtener lecturas de voltaje, este voltaje lo registra el equipo. Trabaja en el rango de -30 a $70{ }^{\circ} \mathrm{C}$ y para un espectro electromagnético de 550 nanómetros. Se ha usado el modelo HW-MJ6558. 
El DHT22 es un sensor digital de temperatura y humedad relativa de buen rendimiento y bajo costo. Utiliza un sensor capacitivo de humedad y un termistor para medir el aire circundante, y muestra los datos mediante una señal digital en la Fig.2: Sensor de temperatura y humedad.

Del pin de datos, se puede obtener nuevos datos una vez cada 2 segundos (solo se utilizará los datos de humedad).

\section{Tarjeta Arduino}

La tarjera Arduino posee 14 entradas/salidas, 6 entradas analógicas, un resonador cerámico de $16 \mathrm{MHz}$, conexión USB, conector de alimentación, etc. La tarjeta contiene todo lo necesario para el funcionamiento del micro controlador; Se conecta al puerto USB o se alimenta con una fuente de voltaje continuo o una batería de 9 VDC para empezar a usarlo.

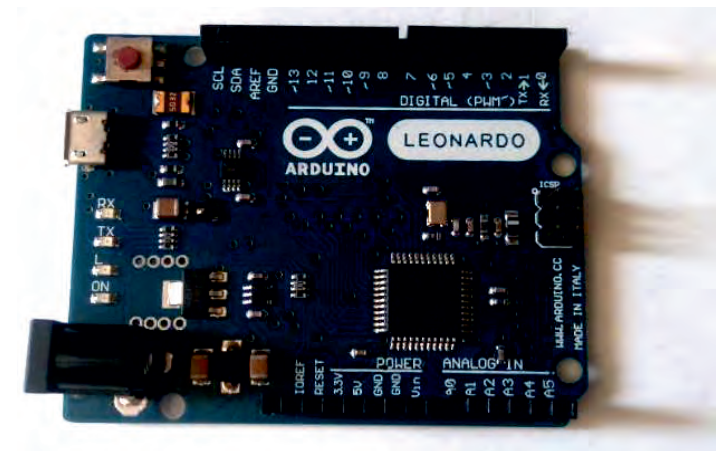

Fig. 3: Tarjeta electrónica Arduino

También se ha usado la tarjeta del reloj calendario, modelo I2C RTC DS3231 AT24C3

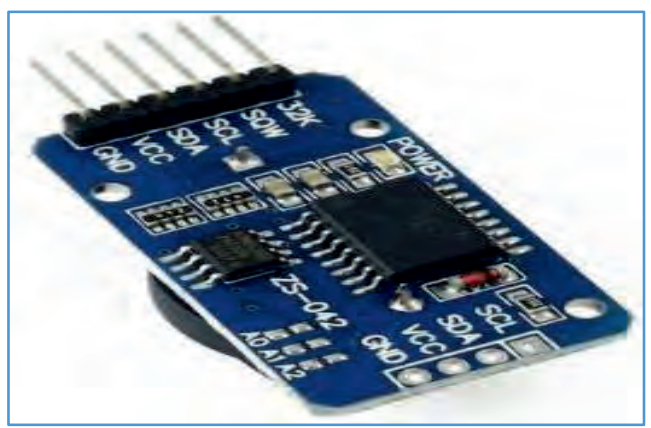

Fig. 4: Tarjeta de reloj calendario

\section{Cubierta}

Los sensores se han colocado en una estructura adaptada que los protege de las condiciones ambientales, pero que permiten la medición de los tres parámetros meteorológicos. Los sensores de temperatura y humedad están dentro mientras que el sensor de Irradiancia está ubicado en la parte externa superior de tal manera que no tiene sombras.

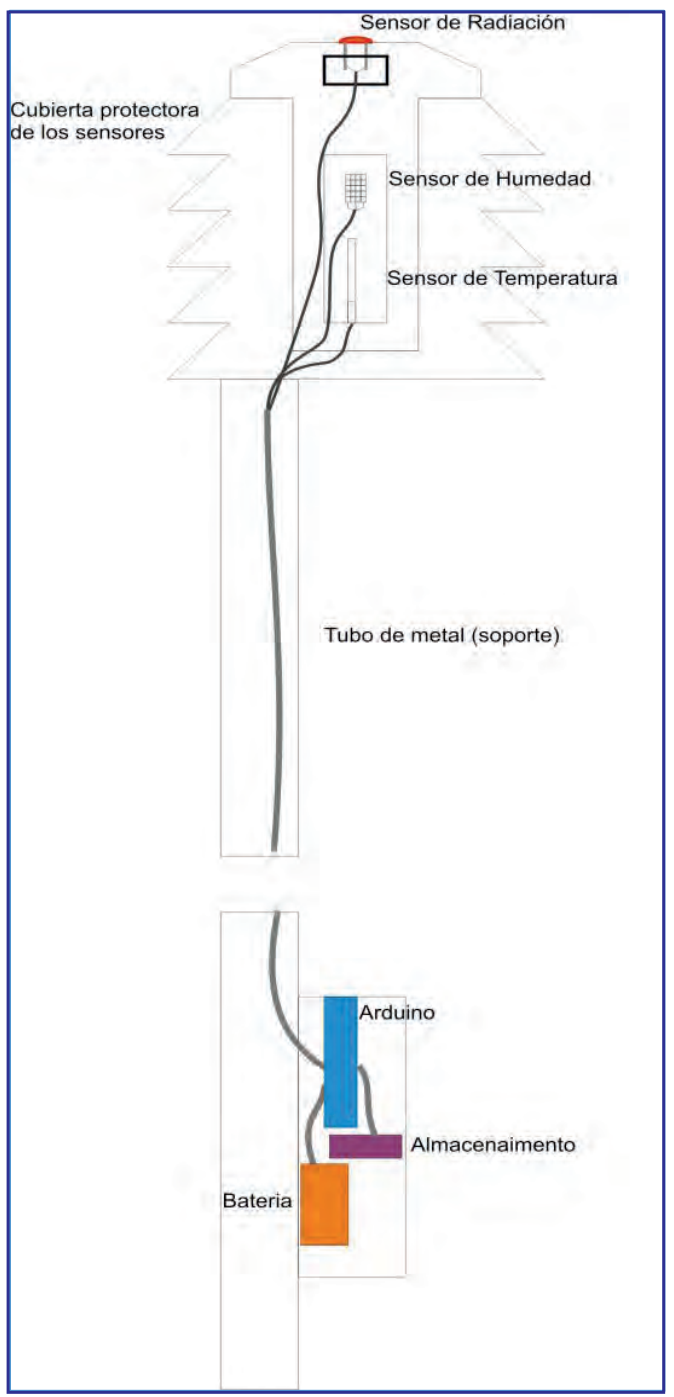

Fig. 5: Esquema de la Estación meteorológica construida

\section{Funcionamiento}

Los sensores envían sus señales a un sistema de adquisición de datos que ha sido construida en base a una placa Arduino, tarjeta de memoria, acondicionadores de señal, reloj, batería, tarjetas prototipadas, elementos electrónicas, cableados, etc. 


\section{Materiales usados en la construcción de la estación}

* 01 Sensor digital de Temperatura modelo DS18B20, $(-20-100)^{\circ} \mathrm{C}$

* 01 Sensor digital de temperatura y humedad relativa modelo DHT22 (0 - 100)\%; (-40 120) ${ }^{\circ} \mathrm{C}$.

- 01 Fotorresistencia tipo LDR, $\varnothing=20$ mm; (631600) ohmios, (300 - 800) nm.

* 01 Tarjeta Arduino, modelo Leonardo.

* 01 módulo SD Card compuesto por una shield SD.

* 01 Reloj calendario modelo I2C RTC DS3231 AT24C3

* 01 Tarjeta SD de $1 \mathrm{~Gb}$

* 01 Resistencia 4,7 k $\Omega$

* 01 Resistencia $10 \mathrm{k} \Omega$

* 03 Resistencia $3 \mathrm{k} \Omega$

* 03 Resistencia $6 \mathrm{k} \Omega$

* 02 Resistencias variable de precisión.

* 02 Resistencia $300 \Omega$ de precisión.

* 01 Resistencia $1 \mathrm{k} \Omega$.

* 01 Circuito integrado OPAM, modelo LM-324

* Cables eléctricos para conexión a computadora y de sensores de $1 \mathrm{~mm}$.

- Conectores DIN y MOLEX (4 entradas)

- Tuercas metálicas de $50 \mathrm{~mm} \times 5 \mathrm{~mm}$

* Tornillos de 1/8; 3,0 mm

* 01 Placa prototipada de $4 \mathrm{~cm} \times 4 \mathrm{~cm}$.

* 01 Batería de 9 VDC, Radio Sharp.

* Base de plástico diseñada en CAD y hecha en impresora 3D.

* Base Metálica o cubierta protectora de aluminio reutilizada.

* 02 pillas de 1,5 VDC para alimentar el sistema.

* Set de herramientas para trabajo eléctrico y mecánico.

- 02 borneras de dos entradas.

* 01 condensador de tantalio de 104 y $22 \mathrm{nF}$.

* 01 regulador de 5 VDC AMS1117

* 02 jumpers.

* 04 abrazaderas de plástico para sujetar los sensores y cables.

* 01 regulador de 9 VDC LT7809.

* 01 carcasa de aluminio para proteger sensores de humedad relativa y temperatura ambiente del viento y de la irradiancia solar, rehusado.

El sistema consta de cuatro etapas que se describen a continuación:

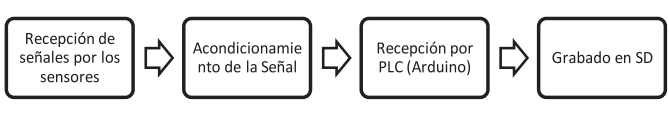

Fig.6: Esquema electrónico de la etapa de grabado de la información en la tarjeta SD.

Los sensores envían información a un micro controlador basado en una tarjeta Arduino el cual recibe la Información, además lee la hora y fecha de la tarjeta de reloj, almacena estos datos en una memoria SD cada 10 segundos.

Fig.7: Sensores ensamblados en la base diseñada y construida

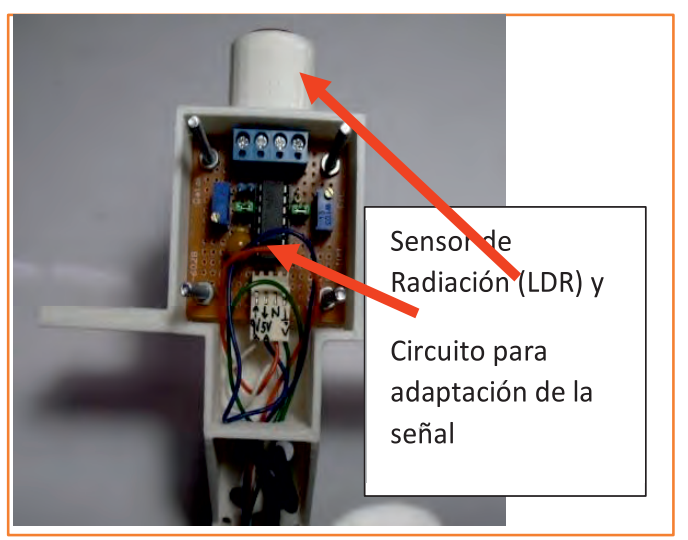

Fig.8: Ubicación de sensores de humedad y temperatura ambiente en la base construida.

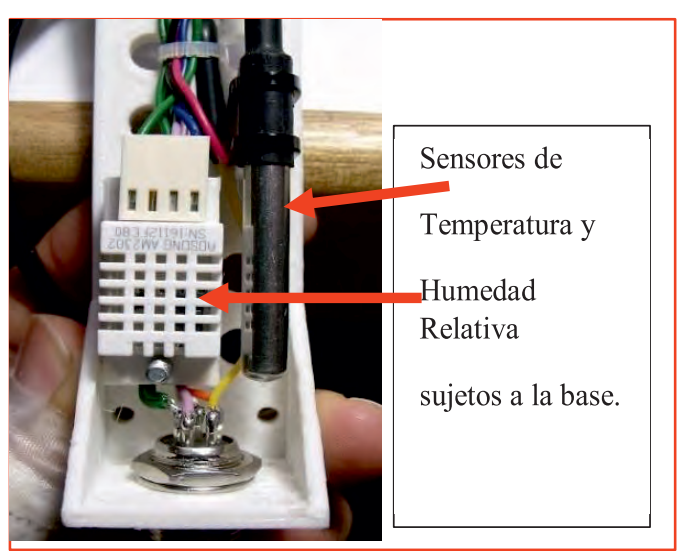




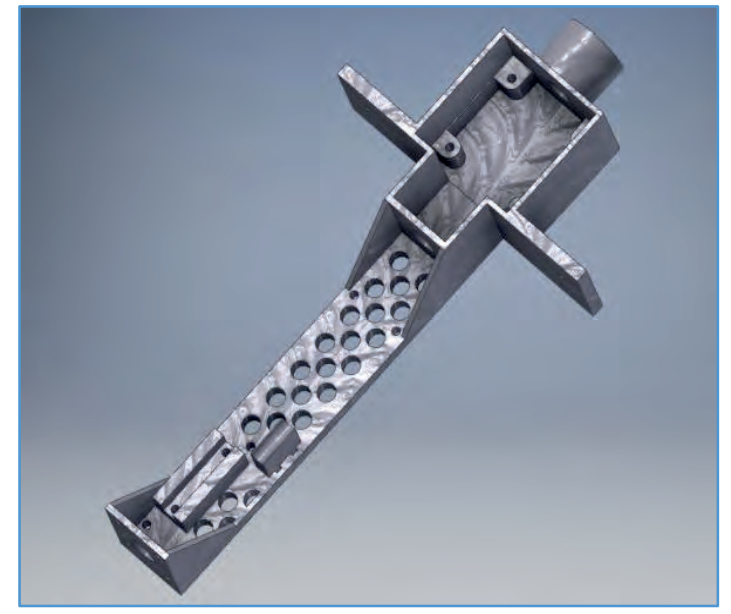

Fig.9: Sistema base para la ubicación y colocación de los sensores de la estación meteorológica, hecho en una impresora en 3D.

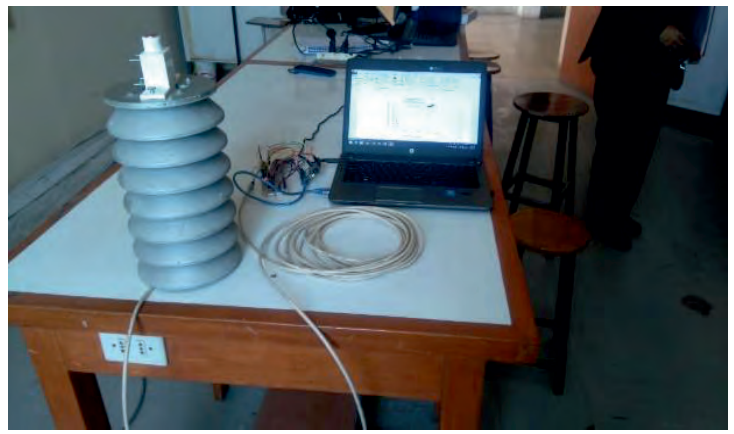

Fig.10: Vista general de la estación meteorológica conectada a la computadora portátil para la transferencia y tratamiento de datos

\section{RESULTADOS EXPERIMENTALES}

\section{ALGORITMO DE PROGRAMACIÓN}

Como la estación está diseñada y construida para registrar continuamente y en forma automática tres parámetros meteorológicos, previamente a la toma y registro de tal información es necesario programarla a través de la tarjeta Arduino y el reloj calendario de acuerdo al intervalo de tiempo que se considere necesario de acuerdo a la investigación a realizar, para nuestro caso se ha programado para que tome datos cada 10 segundos y registre el valor promedio de cada parámetro en un tiempo de un minuto. Así mismo, se ha programado la exactitud de cada uno de lecturas de los parámetros indicados, para la temperatura ambiente de $0,1^{\circ} \mathrm{C}$, humedad relativa $1 \%$ y de la irradiancia solar global de $0,1 \mathrm{~W} / \mathrm{m}^{2}$.

\section{CALIBRACIÓN DEL SENSOR DE IRRADIANCIA LDR:}

El sensor de Irradiancia (LDR acondicionado) ha sido calibrado con un Solarimetro Eppley del Centro de Energías Renovables de Tacna (CERT), con el objetivo de encontrar su constante de calibración a efectos de que conociendo el valor de su resistencia instantánea, podamos inmediatamente conocer el valor de la irradiancia solar global medida por el sensor en Watts $/ \mathrm{m}^{2}$.

Con los datos de la resistencia y los Watts $/ \mathrm{m}^{2}$ se obtiene un gráfico $\left(\mathrm{W} / \mathrm{m}^{2}\right.$ vs resistencia) de cuya pendiente se obtiene la constante de calibración del LDR en Watts $/ \mathrm{m}^{2} \mathrm{x}$ ohmios, de acuerdo a nuestro sistema tenemos el siguiente gráfico:

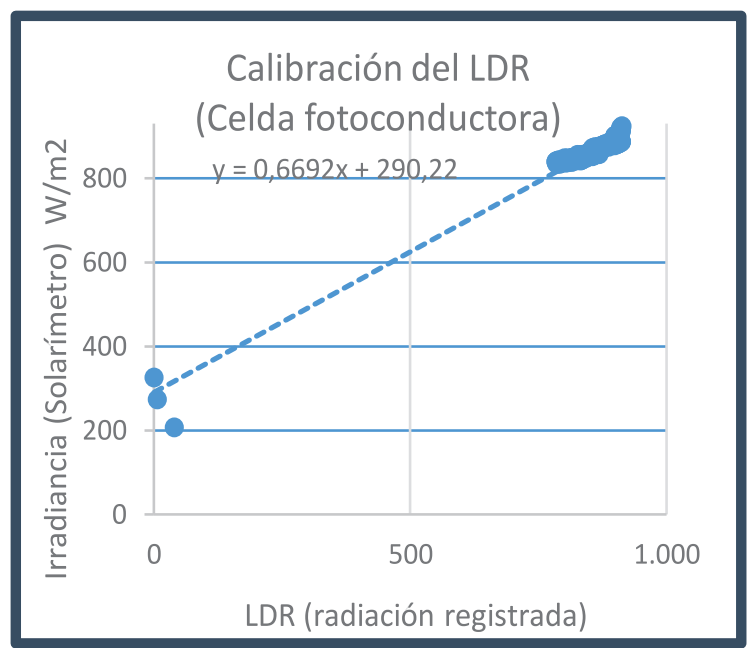

Fig.11: Curva de calibración de la celda fotoconductora

\section{GRÁFICOS EXPERIMENTALES}

A continuación se muestran los gráficos de irradiancia solar global en Watts $/ \mathrm{m}^{2}$, temperatura ambiente en ${ }^{\circ} \mathrm{C}$ y humedad relativa ambiental en $\%$ en función de las horas del día para el periodo del 01 al 02 de abril de 2017.

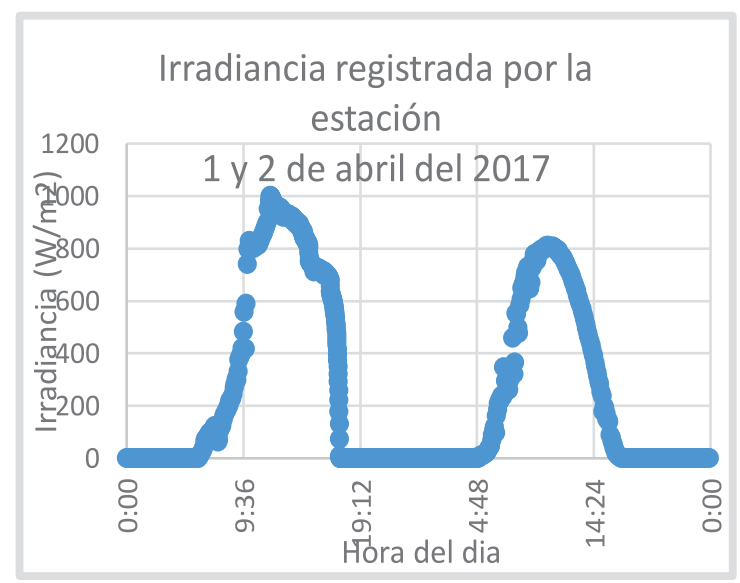


Fig.12: Curva de irradiancia solar global sobre una superficie horizontal obtenida con datos registrados con el sensor LDR de la estación meteorológica

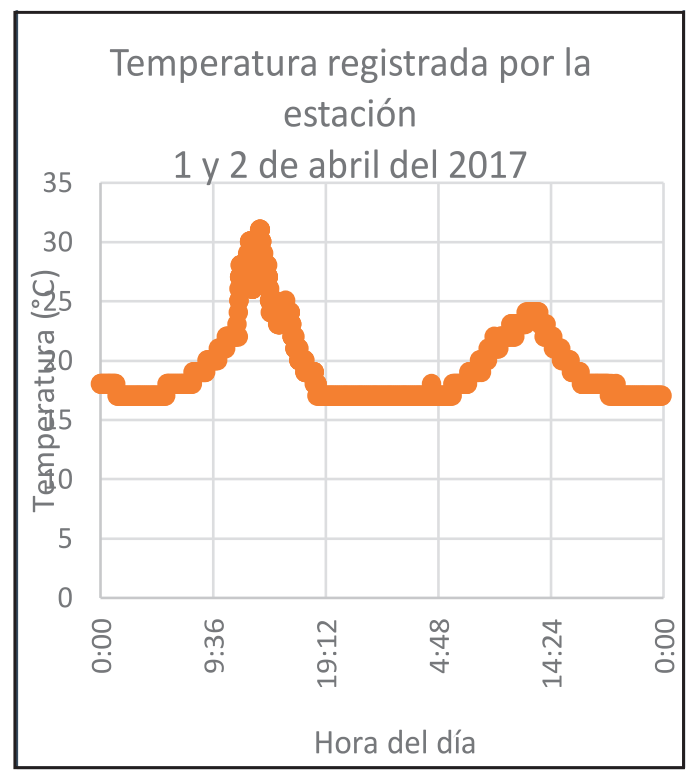

Fig.13: Curva interdiaria de la temperatura ambiente obtenida con información de la estación meteorológica

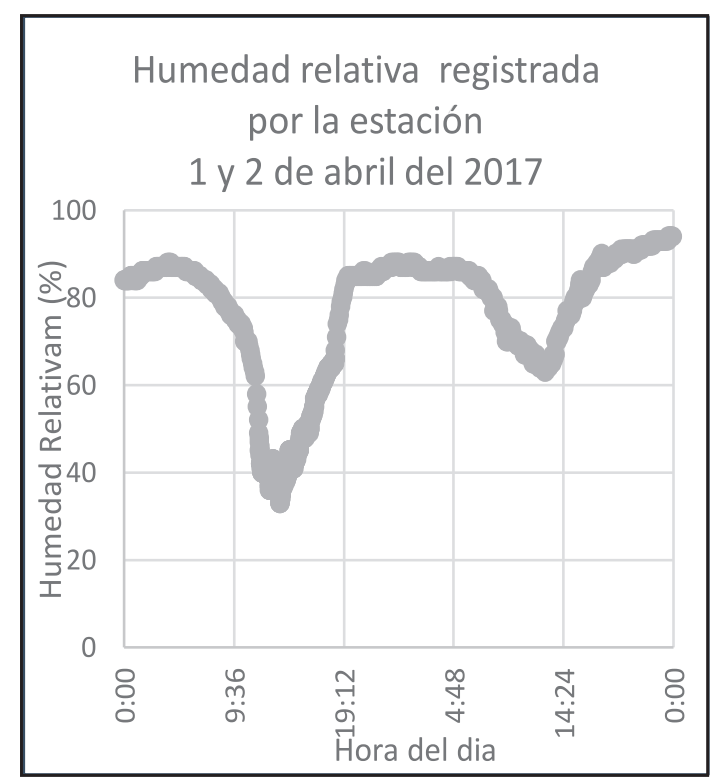

Fig. 14: Curva de variación interdiaria de la humedad relativa ambiente obtenida con información registrada por la estación meteorológica

\section{CONCLUSIONES}

$\checkmark$ Se ha logrado diseñar, construir y sistematizar una mini estación meteorológica automática para la medida de tres parámetros ambientales: Irradiancia solar global, temperatura y humedad relativa ambiental, con precisiones de $0,1 \mathrm{~W} / \mathrm{m}^{2}, 0,1^{\circ} \mathrm{C}$ y $1 \%$, respectivamente, usando sensores adquiridos en el mercado nacional $y$ local.

$\checkmark$ Para el registro, almacenamiento, programación del sistema y procesamiento de la información se ha usado el hardware libre ARDUINO.

$\checkmark \quad$ La información registrada por la estación es en tiempo real, para esto se transfieren a una computadora para el proceso de los datos correspondientes con los cuales se obtienen los gráfico de las parámetros en función del tiempo.

$\checkmark$ Los autores del trabajo han desarrollado un "NOW - HOW" para el diseño, construcción y sistematización de estaciones meteorológicas de bajo costo y de buena funcionabilidad, aspecto que debe mejorarse en futuros trabajos similares.

$\checkmark$ Con el conocimiento desarrollado, la mini estación meteorológica puede ser más integral para monitorear otros parámetros meteorológicos de interés para el estudio de la influencia del cambio climático y prevención de desastres naturales en la región.

\section{REFERENCIAS BIBLIOGRÁFICAS}

Actas del congreso: 3rd International Conference on Experiences with Automatic Weather Stations, 2003.

BOYLESTAD, R. L. Y NASHELSKY, L. (2009). Electrónica: Teoría de circuitos y dispositivos electrónicos, décima edición, Editorial Pearson Educación, México.

COUGHLIN, R. F. Y DRISCOLL, F. F. (1991). Operational amplifiers and linear integrated circuits, Cuarta edición, Editorial Prentice Hall. Englewood Cliffs N.J.

COULSON, K. L. (1975). Solar and terrestrial radiation: Methods and measurements. Editorial Academic Press, New York

DUFFIE, J. A. Y BECKMAN, W. A. (2013). Solar engineering of thermal processes, Cuarta edición, Editorial John Wiley, Hoboken, Canada ENERGY, S. (1992). Calibration of field pyranometer by comparison to a reference pyranometer, ISO, India

GARG, H. P. Y GARG, S. N. (1993). Measurement of solar radiation I. Radiation instruments, Renewable energy, 3(4-5), 321-333, New Delhi, India 
Guide to meteorological instruments and methods of observation, Setima edición (2008). WMO-[publication]: N 8, Edición 7. Geneva, Switzerland

IQBAL, M. (1983). An introduction to solar radiation, New York: Academic Press, Toronto,

KLUGMANN, E. Y ONYEOGU, O. E. (1979). Measurement of insolation using $\mathrm{CdS}$ photoresistor, Energy Conversion, 19(3), 153-157, Nigeria

MARGOLIS, M. (2011). Arduino cookbook. Farnham, O'Reilly, Beijing, England

MEDUGU, D. W., BURARI, F. W. Y ABDULAZEEZ, A. A. (2010). Construction of a reliable model pyranometer for irradiance measurements. African Journal of Biotechnology, Nigeria

MYERS, D. (2013). Solar radiation: Practical modeling for renewable energy applications, Energy and the environment. Boca Raton: CRC Press/Taylor \& Francis Group. USA

OKEKE, C. E. Y VARKEY, A. J. (1981). Measurement of insolation using $\mathrm{CdS}$ photoresistor, Energy Conversion and Management, 21(4), 267-273, Nigeria

ŞEN, Z. (2008). Solar energy fundamentals and modeling techniques: Atmosphere, environment, climate change, and renewable energy. Editorial Springer. London

TWIDELL, J. Y WEIR, A. D. (2006). Renewable energy resources, Segunda edición, Editorial Taylor \& Francis. London, New York:

VIGNOLA, F., MICHALSKY, J. Y STOFFEL, T. L. (2012). Solar and infrared radiation measurements, Energy and the environment, Editorial CRC Press. . Boca Raton FL

ROSA MARÍA RODRÍGUEZ JIMÉNEZ, ÁGUEDA BENITO CAPA, ADELAIDA PORTELLA LOZANO. Meteorología y Climatología. Fundación Española para la Ciencia y la Tecnología, Madrid, 2006

[19]ZEKAI SEN: Solar Energy Fundamentals and Modeling Techniques, Editorial Springer, Universidad Technical of Estambul, 2008

EDUARDO LORENZO, Electricidad Solar, Editorial Progensa, España 2000

PETER WURFEL, Physics of Solar Cells, from Principles to new Concepts, University
Karlsruhe, Editorial Wiley-VCH, Weinheim, Alemania 2005

Organización Meteorológica Mundial (OMM). 1 992. Vocabulario meteorológico internacional. OMM N¹82, Suiza.

J. A. CHASSERIAUX, Conversión Térmica de la Radiación Solar, Librería Agropecuaria S.A., Primera Edición, Argentina, 1990.

JEREMY BLUM, Exploring Arduino, Editrial Wiley, Cánada 2013

MICHAEL MARGOLIS, ARDUINO COOKBOOK, Editorial OReilly, USA 2011

[26]POLO BRAVO, CARLOS: (2010) EI potencial energético renovable de la región Tacna, XIX Simposio Peruano de Física, Universidad Nacional de Ingeniería (UNI), Lima, Agosto del 2010; IV Conferencia Latinoamericana de Energía Solar, y XVII Simposio Peruano de Energía Solar; International Society Energy Solar (ISES), (APES), Universidad Nacional de San Antonio Abad del Cusco (UNSAAC), Cusco, Noviembre del 2010.

POLO BRAVO C., TORRES MURO H., BERNABÉ COAQUERA R., GUERRERO FARRO A., MONITOREO, Tratamiento y Sistematización de Parámetros Meteorológicos de la ciudad de Tacna, Informe COIN marzo 2016, Tacna, Perú.

GOL, A.W., Instrumentos Meteorológicos, Vol III, 2da. Edición, Servicio de Meteorología y Comunicaciones, República de Venezuela, 1964.

MADRID ANTONIO (2009), Energías Renovables: Fundamentos, Tecnologías y Aplicaciones; primera edición; AMV Ediciones, Mundi Prensa; Madrid, España

J.TWIDELL, A. WEIR, Renewable Energy Recurces, Chapman and Hall, USA, 1990.

OMM, WMO, PNUE, UNEP: Grupo Intergubernamental de expertos sobre el Cambio Climático (IPCC); 1997, 2000, 2008; Informe Especial del IPCC: Impactos Regionales del cambio Climático

NOAA. 2002. Sunrise / Sunset Calculator. Surface Radiation Research Branch. Department of Commerce, USA [On line]. http://www.srrb.noaa.gov/hightlights/sunrise/su nrise.html 